\title{
Characterizations of Certain Doubly Truncated Distribution Based on Order Statistics
}

\section{Shawky $\mathrm{Al}^{1 *}$ and Badr $\mathrm{MM}^{2}$}

${ }^{1}$ Department of Statistics, Faculty of Science, King Abdulaziz University, Saudi Arabia

${ }^{2}$ Department of Statistics, Faculty of Science for Girls, King Abdulaziz University, Saudi Arabia

\begin{abstract}
In this paper, we characterize doubly truncated classes of absolutely continuous distributions by considering the conditional expectation of functions of order statistics. Specific distributions considered as a particular case of the general class of distributions are Weibull, Pareto, Power function, Rayleigh and Inverse Weibull.
\end{abstract}

Keywords: Double truncated; Order statistics; Conditional expectation; Weibull, Pareto; Rayleigh; Inverse Weibull distributions

AMS 2000 Subject Classification: 62E10; 62G30

\section{Introduction}

The order statistics arise naturally in many real life applications and it is considered as an increasingly important subject. Articles relating to this area have appeared in numerous different publications. Many authors have studied order statistics; for example, David [1], Balakrishnan and Cohen [2], Arnold et al. [3], David [4], David and Nagaraja [5] and Mahmoud et al. [6,7]. Several authors discussed conditional expectations, for example, Balakrishnan and Sultan [8], Mohie El-Din et al. [9], Abu-Youssef [10], Abd- El-Mougod [11], Shawky and Abu-Zinadah [12], Shawky and Bakoban [13] and Pushkarna et al. [14].

Let $X_{1: n} \leq X_{2: n} \leq \ldots \leq X_{n: n}$ be the first $n$ order statistics based on distribution with probability density function (pdf) $\mathrm{f}(\mathrm{x})$ and cumulative distribution function (cdf) $\mathrm{F}(\mathrm{x})$. Then the pdf of the $\mathrm{r}^{\text {th }}$ order statistics, $X_{r: n}, 1 \leq r \leq n$, is given by (see David (1981))

$$
f_{r}(x)=C_{r}[F(x)]^{r-1}[1-F(x)]^{n-r} f(x),
$$

where $C_{r}=\frac{n !}{(r-1) !(n-r) !},-\infty<x<\infty$,

and the joint pdf of two order statistics $X_{r: n}$ and $X_{s: n}, 1 \leq r \leq \mathrm{s} \leq n$ is given by

$$
f_{r, s}(x, y)=C_{r, s}[F(x)]^{r-1}[F(y)-F(x)]^{s-r-1}[1-F(y)]^{n-s} f(x) f(y),-\infty<x<y<\infty,
$$

where $C_{r, s}=\frac{n !}{(r-1) !(s-r-1) !(n-s) !}$.

The doubly truncated case of a distribution is the most general case since it includes the right truncated, left truncated and non-truncated distributions as special cases, Joshi [15], Balakrishnan and Joshi [16], Khan and Ali [17] and Ahmad [18], among others, investigated doubly truncated distributions.

Suppose that the random variable $\mathrm{X}$ has a $\mathrm{cdf} F(\mathrm{x})$ and $\mathrm{pdf} f(\mathrm{x})$, where $\alpha \leq x \beta$. Let, for given $\varepsilon$ and

$$
\int_{\alpha}^{\varepsilon} f(x) d x=F(\varepsilon)=P \text { and } \int_{\alpha}^{\gamma} f(x) d x=F(\gamma)=Q .
$$

Then the doubly truncated pdf of X, say $\mathrm{g}(\mathrm{x})$, and cdf, say $\mathrm{G}(\mathrm{x})$, are given respectively by

$$
g(x)=\frac{f(x)}{l_{1}}, \alpha \leq \varepsilon<x<\gamma \leq \beta,
$$

$$
G(x)=\frac{F(x)-P}{l_{1}}, \varepsilon<x<\gamma,
$$

where

$$
l_{1}=Q-P, G(\varepsilon)=0 \text { and } G(\gamma)=1 .
$$
[3] by

The conditional density function of $X_{s: n} y$, given that $X_{r, n=} x$ is given

$$
\begin{aligned}
& f_{S \mid r}\left(y \mid X_{r: n}=x\right)=\frac{(n-r) !}{(s-r-1) !(n-s) ![1-G(x)]^{n-r}} . \\
& {[G(y)-G(x)]^{S-r-1}[1-G(y)]^{n-S} g(y),} \\
& \varepsilon<x<y<\gamma .
\end{aligned}
$$

Also, the conditional density function of $X_{r: n=} x$, given that $X_{s: n} y$ is given by

$$
\begin{aligned}
& f_{r \mid S}\left(x \mid X_{S: n}=y\right)=\frac{(s-1) !}{(r-1) !(s-r-1) ![G(y)]^{s-1}} . \\
& {[G(x)]^{r-1}[G(y)-G(x)]^{S-r-1} g(x)}
\end{aligned}
$$

Let

$$
\mu_{s \mid r}=E\left[\varphi\left(X_{s: n}\right) \mid X_{r: n}=x\right] \text { and } \mu_{r \mid s}=E\left[\varphi\left(X_{r: n}\right) \mid X_{s: n}=y\right],
$$

where $\varphi($.$) is a monotonic, continuous and differentiable function on$ the interval $(\alpha, \beta)$. For abbreviation, we will denote

$$
\mu_{S \mid r}=E_{S \mid r}\left[\varphi(Y) \mid X_{r n}=x\right] \text { and } \mu_{r \mid S}=E_{r \mid S}\left[\varphi(X) \mid X_{S: n}=y\right] .
$$

\section{Main Results}

In this section, we characterize three general classes of distributions,

$$
F(x)=1-\left[b-a e^{-\varnothing(x)}\right]^{c}, \alpha<x<\beta \text {, i.e., }
$$

*Corresponding author: Shawky Al, Department of Statistics, Faculty of Science King Abdulaziz University, P.O. Box 80203, Jeddah 21589, Saudi Arabia, Tel: +966 800116 9528; E-mail: aishawky@yahoo.com

Received October 24, 2016; Accepted November 23, 2016; Published November 29, 2016

Citation: Shawky Al, Badr MM (2016) Characterizations of Certain Doubly Truncated Distribution Based on Order Statistics. J Appl Computat Math 5: 329. doi: 10.4172/2168-9679.1000329

Copyright: (c) 2016 Shawky Al, et al. This is an open-access article distributed under the terms of the Creative Commons Attribution License, which permits unrestricted use, distribution, and reproduction in any medium, provided the original author and source are credited. 


$$
G(x)=\frac{1}{l}\left\{\left[b-a e^{-\varnothing(x)}\right]^{c}-v\right\}, \varepsilon<x<\gamma,
$$

where

$$
v=1-P, l=P-Q, P=F(\varepsilon), Q=F(\gamma), G(\varepsilon)=0, G(\gamma)=1,
$$

$F(\alpha)=0, F(\beta)=1$, and $a \neq 0, c 0, b$ are finite constants.

$F(x)=1-[a-b \emptyset(x)]^{c}, \alpha<x<\beta$, i.e.,

$$
G(x)=\frac{1}{l}\left\{[a-b \varnothing(x)]^{c}-v\right\}, \varepsilon<x<\gamma,
$$

where

$v=1-P, l=P-Q, P=F(\varepsilon), Q=F(\gamma), G(\varepsilon)=0, G(\gamma)=1$,

$F(\alpha)=0, F(\beta)=1$, and $b \neq 0, c \neq 0, a$ are finite constants.

$$
\begin{aligned}
& F(x)=1-\left[b-a e^{-C \varnothing(x)}\right], \alpha<x<\beta, \text { i.e., } \\
& G(x)=\frac{1}{l}\left\{\left[b-a e^{-C \varnothing(x)}\right]-v\right\}, \varepsilon<x<\gamma,
\end{aligned}
$$

where

$v=1-P, \mathrm{l}=P-Q, P=F(\varepsilon), Q=F(\gamma), G(\varepsilon)=0, G(\gamma)=1$,

$F(\alpha)=0, F(\beta)=1$, and $C \neq 0, a>0, b>0$ are finite constants.

Note: If we put $l=-1, v=1$, thus $\mathrm{G}(\mathrm{x})$ reduces to complete cdf of $\mathrm{x}$, i.e. $\mathrm{F}(\mathrm{x}), \alpha<x<\beta$.

Let $\mathrm{X}$ be an absolutely continuous random variables with pdf $\mathrm{g}(\mathrm{x})$, cdf $\mathrm{G}(\mathrm{x})$ and $\emptyset(x)$ is a monotonic, continuous and differentiable function on $(\varepsilon, \gamma)$.

Theorems 1-4 given below characterize the general class given by (2.1), Theorems 5-8 characterize the general class given by (2.2), while Theorems 9-12 characterize the general class given by (2.3).

\section{Theorem 1}

Referring to (1.6), (1.7) and (2.1), then

$\mu_{r \mid s}=\mu_{r+|| s}+\frac{1}{a c r}\left\{a E_{r \mid s}\left[V(X) \mid X_{s: n}=y\right]-b E_{r \mid s}\left[V(X) e^{\varphi(X)} \mid X_{r: s}=y\right]\right\}$,

where

$V(x)=\frac{l G(x)}{l G(x)+v}$.

\section{Proof}

It is clear from (1.6) and (1.7) that

$\mu_{r \mid s}=\frac{(s-1) !}{(r-1) !(s-r-1) ![G(y)]^{s-1}} \int_{\varepsilon}^{y} \varphi(x)[G(x)]^{r-1}[G(y)-G(x)]^{s-r-1} g(x) d x$.

Integrating (2.6) by parts, we get

$$
\mu_{r \mid s}=\mu_{r+|| s}-\frac{(s-1) !}{r !(s-r-1) ![G(y)]^{s-1}} \int_{\varepsilon}^{y} \varphi(x)[G(x)]^{r}[G(y)-G(x)]^{s-r-1} d x .
$$

Differentiating (2.1) with respect to $\mathrm{x}$, we have

$$
\varphi(x)=\frac{b e^{\varphi(x)}-a}{a c} \frac{\lg (x)}{l G(x)+v} .
$$

From (2.7) and (2.8), we obtain

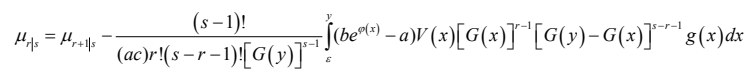

$$
\begin{aligned}
& =\mu_{r+1 \mid s}-\frac{1}{a c r} \int_{\varepsilon}^{y}\left[b e^{g(x)}-a\right] V(x) g_{r k}(x \mid y) d x \text {. }
\end{aligned}
$$

Simplifying (2.9), we get (2.4). Thus, the theorem is proved.

\section{Theorem 2}

Referring to (1.6), (1.7), then (2.1) if and only if

$$
\begin{aligned}
& \mu_{r \mid r+1}=\varphi(y)+\frac{1}{a c} \sum_{i=0}^{\infty}(-1)^{i+1}\left(\frac{l}{v}\right)^{i+1} \\
& \frac{[G(y)]^{i+1}}{r+i+1}\left\{b E_{r+i+1 \mid r+i+2}\left[e^{\varphi\left(X_{r+i+1: n}\right)} \mid X_{r+i+2: n}=y\right]-a\right\}
\end{aligned}
$$

\section{Proof}

It is clear that

$$
\mu_{r \mid r+1}=\frac{r}{[G(y)]^{r}} \int_{\varepsilon}^{y} \varphi(x)[G(x)]^{r-1} g(x) d x .
$$

Integrating by parts, we get

$$
\mu_{r \mid r+1}=\varphi(y)-\frac{1}{[G(y)]^{r}} \int_{\varepsilon}^{y} \varphi(x)[G(x)]^{r} d x
$$

Compensation for (2.8) in (2.11), we have

$$
\mu_{r \mid r+1}=\varphi(y)-\frac{l}{a c[G(y)]^{r}}\left\{\int_{\varepsilon}^{y}\left(b e^{\varphi(x)}-a\right) \frac{\mathrm{g}(x)}{l G(x)+v}[G(x)]^{r} d x .\right.
$$

Expand $\frac{1}{l G(x)+v}$ and compensation for (2.12), after some simplification, we get (2.10). Thus (2.1) implies (2.10). Now from (1.6) and (2.10), we obtain

$$
\begin{aligned}
& \frac{r}{[G(y)]^{r}} \int_{\varepsilon}^{y} \varphi(x)[G(x)]^{r-1} g(x) d x= \\
& \varphi(y)+\frac{1}{a c} \sum_{i=0}^{\infty}(-1)^{i+1}\left(\frac{l}{v}\right)^{i+1} \frac{[G(y)]^{i+1}}{r+i+1}\left\{b \int_{\varepsilon}^{y} e^{\varphi(x)}[G(x)]^{r+i} g(x) d x-a\right\}
\end{aligned}
$$

Taking the derivative, we get

$$
\varphi(y)=\frac{\left(\frac{l}{v}\right) g(y)}{1+\frac{l}{v} G(y)}\left[\frac{b}{a c} e^{\varphi(y)}-\frac{1}{c}\right],
$$

which gives

$$
\frac{a c \varphi(y) e^{-\varphi(y)}}{b-a e^{-\varphi(y)}}=\frac{\lg (y)}{v+l G(y)} .
$$

Integrate (2.14), hence $\mathrm{G}(\mathrm{y})$ has the form (2.1), and so (2.10) implies (2.1).

\section{Special case:}

Return to the (2.10), if we put $l=-1, v=1$ we get

$$
\begin{aligned}
& \mu_{r \mid r+1}=\varphi(y)+\frac{1}{a c} \sum_{i=0}^{\infty} \frac{[F(y)]^{i+1}}{i+r+1}\left\{b E_{r+i+1 \mid r+i+2}\left[e^{\varphi\left(X_{r+i+t+n}\right)} \mid X_{r+i+2: n}=y\right]-a\right\}, \\
& \alpha<x<y<\beta
\end{aligned}
$$

the relation (2.15) is before doubly truncated case.

\section{Theorem 3}

Referring to (1.5), (1.7) and (2.1), then

$$
\begin{aligned}
& \mu_{s \mid r}=\mu_{s-1 \mid r}+\frac{l}{a c(n-s+1)} \\
& \left\{a E_{s \mid r}\left[N\left(X_{s: n}\right) \mid X_{r: n}=x\right]-b E_{s \mid r}\left[e^{\varphi\left(X_{s n}\right)} N\left(X_{s: n}\right) \mid X_{r: n}=x\right]\right\}
\end{aligned},
$$


where

$$
N(y)=\frac{[1-G(y)]}{[l G(y)+v]} .
$$

It is clear from (1.5) and (1.7) that

$\mu_{s \mid r}=\frac{(n-r) !}{(s-r-1) !(n-s) ![1-G(x)]^{n-r}} \int_{x}^{y} \varphi(y)[G(y)-G(x)]^{s-r-1}[1-G(y)]^{n-s} g(y) d y$.

Integrating by parts, we get

$\mu_{s \mid r}=\mu_{s-1 \mid r}-\frac{(n-r) !}{(s-r-1) !(n-s+1) ![1-G(x)]^{n-r}} \int_{x}^{\gamma}$.

$\varphi(y)[G(y)-G(x)]^{s-r-1}[1-G(y)]^{n-s+1} d y$

Substituting (2.7) in (2.18), we get

$\mu_{s \mid r}=\mu_{s-|| r}-\frac{(n-r) !}{(s-r-1) !(n-s+1) ![1-G(x)]^{n-r}} \int_{x}^{\gamma} \frac{e^{\varphi(y)}}{a c}\left[b-a e^{-\varphi(y)}\right] N(y)[G(y)-G(x)]^{s-r-1}$

$\mu_{s \mid r}=\mu_{s-|| r}-\frac{(n-r) !}{(s-r-1) !(n-s+1) ![1-G(x)]^{n-r}} \int_{x}^{\gamma} \frac{e^{\varphi(y)}}{a c}\left[b-a e^{-\varphi(y)}\right] N(y)[G(y)$

$-G(x)]^{\mathrm{s}^{-r-1}} \times[1-G(y)]^{n-s} d y$

$=\mu_{s-|| r}+\frac{l}{a c(n-s+1)}\left\{\int\left[a-b e^{\varphi(y)}\right] N(y) g_{s \mid r}(y \mid x) d y\right.$

After some simplification, we get (2.16).

\section{Theorem 4}

Referring to (1.5), (1.7), then (2.1) if and only if

$\mu_{r+1 \mid r}=\varphi(x)+\frac{l b}{a c(n-r)} E_{r+1 \mid r}$

$\left[N(y) e^{\varphi(y)} \mid X_{r}=x\right]-\frac{l}{c(n-r)} E_{r+1 \mid r}\left[N(y) \mid X_{r: n}=x\right]$

where $N(y)$ is defined in (2.17).

\section{Proof}

It is clear that

$\mu_{r+1 \mid r}=\frac{(n-r)}{[1-G(x)]^{n-r}} \int_{x}^{\gamma} \varphi(y)[1-G(y)]^{n-r-1} g(y) d y$.

Integrating by parts, we get

$\mu_{r+|| r}=\varphi(x)+\frac{1}{[1-G(x)]^{n-r}} \int_{x}^{\gamma} \varphi(y)[1-G(y)]^{n-r} d y$.

Compensation for (2.8) in (2.22), we have

$$
\mu_{r+1 \mid r}=\varphi(x)+\frac{l}{a c[1-G(x)]^{n-r}} \int_{x}^{\gamma} \frac{b e^{\varphi(y)}-a}{[l G(y)+v]} \cdot[1-G(y)]^{n-r} g(y) d y .
$$

Simplifying (2.23), we obtain (2.20). Thus (2.1) implies (2.20), i.e. the necessary condition is proved. To prove the sufficient condition, from (2.20) and (1.7), we have

$$
\begin{aligned}
& \frac{(n-r)}{[1-G(x)]^{n-r}} \int_{x}^{r} \varphi(y)[1-G(y)]^{n-r-1} g(y) d y=\varphi(x)+\frac{l b}{a c[1-G(x)]^{n-r}} \int_{x}^{e^{\varphi(y)}} \\
& \times[1-G(y)]^{n-r-1} N(y) g(y) d y-\frac{l}{c[1-G(x)]^{n-r}} \int_{x}^{r} N(y)[1-G(y)]^{n-r-1} g(y) d y
\end{aligned}
$$

Taking the derivative of (2.24) with respect to $\mathrm{x}$, we get (2.8), and integrate it we have (2.1), thus (2.20) implies (2.1). Then, the Theorem is proved.

\section{Special case}

Return to (2.17), if we put $l=-1, v=1$ we get

$$
\mu_{r+1 \mid r}=\varphi(x)-\frac{b}{a c(n-r)} E_{r+1 \mid r}\left[e^{\varphi(y)} \mid X_{r}=x\right]+\frac{1}{c(n-r)}, \alpha<x<y<\beta,
$$

it is before doubly truncated case (Table 1).

\section{Theorem 5}

Referring to (1.6), (1.7) and (2.2), then

$\mu_{r \mid s}=\mu_{r+1 \mid s}-\frac{1}{b c r}\left\{b E_{r \mid s}\left[\varphi\left(X_{r=n}\right) V\left(X_{r \cdot n}\right) \mid X_{s, n}=y\right]-a E_{r \mid s}\left[V\left(X_{r n}\right) \mid X_{s, n}=y\right]\right\}$,

where $V(x)$ is defined in (2.5).

\section{Proof}

As before in Theorem (1), differentiate (2.2) with respect to $x$, we have

$$
\varphi(x)=\frac{b \varphi(x)-a}{b c} \frac{\lg (x)}{l G(x)+v} .
$$

\begin{tabular}{|c|c|c|c|c|}
\hline Name & {$[I G(x)+v]$} & $\varphi(x)$ & $(I, v)$ & $(a, b, c)$ \\
\hline Weibull & $e^{-\theta x^{p}} ; \alpha \leq \varepsilon<x<\gamma \leq \beta, \varepsilon=0, \gamma \rightarrow \infty$ & $\begin{array}{l}X^{p} \\
\theta x^{p}\end{array}$ & $\left(e^{-\theta \varepsilon^{p}}-e^{-\theta \gamma^{p}}, e^{-\theta \varepsilon^{p}}\right)$ & $\begin{array}{l}(-1,0, \theta) \\
(-1,0,1)\end{array}$ \\
\hline Pareto & $\theta^{o} x^{p} ; \alpha \leq \varepsilon<x<y \leq \beta, \varepsilon=0, \gamma \rightarrow \infty$ & $\begin{array}{l}\ln (x) \\
\ln \left[x^{-p}\right]\end{array}$ & $\left(\theta^{p}\left(\gamma^{-p}-\varepsilon^{-p}\right), \theta^{p} \varepsilon^{-p}\right)$ & $\begin{array}{l}(-\theta, 0, p) \\
\left(-\theta^{p}, 0,-1\right)\end{array}$ \\
\hline Power function & $1-\theta^{-p} x^{p}, \alpha \leq \varepsilon<x<y \leq \beta, \varepsilon=0, y \rightarrow \infty$ & $\begin{array}{l}\ln \left[\frac{x}{\theta}\right]^{-p} \\
\ln \left[x^{p}\right]\end{array}$ & $\left(\theta^{-p}\left(\varepsilon^{p}-Y^{p}\right), 1-\theta^{-p} \varepsilon^{p}\right)$ & $\begin{array}{l}(1,1,1) \\
\left(\theta^{-p}, 1,1\right)\end{array}$ \\
\hline Rayleigh & $e^{\left(-\theta x^{2}\right)} ; a \leq \varepsilon<x<\gamma \leq \beta$ & $x^{2}$ & $\left(e^{-\theta \gamma^{2}}+e^{-\theta \varepsilon^{2}}, e^{-\theta \gamma^{2}}\right)$ & $(-1,0, \theta)$ \\
\hline Inverse Weibull & $e^{\left(-\theta x^{-p}\right)} ; a \leq \varepsilon<x<\gamma \leq \beta$ & $\theta x^{-p}$ & $\left(e^{-\theta \gamma^{-p}}+e^{-\theta \varepsilon^{-p}}, e^{-\theta \varepsilon^{-p}}\right)$ & $(-1,0,1)$ \\
\hline
\end{tabular}

Compensation for (2.26) in (2.7), we get

$$
\begin{aligned}
& \mu_{r \mid s}=\mu_{r+1 \mid s}-\frac{(s-1) !}{r !(s-r-1) ![G(y)]^{s-1}} \int_{\varepsilon}^{y}\left\{\frac{b \varphi(x)-a}{b c}\right\} \\
& V(x)[G(x)]^{r-1}[G(y)-G(x)]^{s-r-1} g(x) d x \\
& =\mu_{r+1 \mid s}-\frac{1}{b c r} \int_{\varepsilon}^{y}(b \varphi(x)-a) V(x) g_{r \mid s}(x \mid y)^{r-1} d x
\end{aligned}
$$

Simplifying (2.27), we obtain (2.25). Thus, the Theorem is proved.

Table 1: Example of $G(x)=\frac{1}{l}\left\{\left[b-a e^{-\varphi(x)}\right]^{c}-v\right\}$ distributions. 


\section{Theorem 6}

Referring to (1.6), (1.7), then (2.2) if and only if

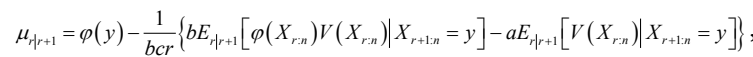

Where $V(x)$ is defined in (2.5).

\section{Proof}

As before in Theorem (2), from (2.26) and (2.11), we have

$$
\begin{aligned}
& \mu_{r \mid r+1}=\varphi(y)-\frac{1}{[G(y)]^{r}} \int_{\varepsilon}^{y} \frac{b \varphi(x)-a}{b c} \frac{l G(x)}{l G(x)+v}[G(x)]^{r-1} g(x) d x \\
& =\varphi(y)-\frac{1}{b c r} \int_{\varepsilon}^{y}(b \varphi(x)-a) V(x) g_{r \mid r+1}(x \mid y) d x
\end{aligned}
$$

Therefore, we get (2.28), then (2.2) implies (2.28). To prove the sufficient condition, from (2.28) and (1.7), we obtain

$$
\begin{aligned}
& \frac{r}{[G(y)]^{r}} \int_{\varepsilon}^{y} \varphi(x)[G(x)]^{r-1} g(x) d x=\varphi(y)- \\
& \frac{1}{b c r} \int_{\varepsilon}^{y}(b \varphi(x)-a) \frac{l G(x)}{l G(x)+v}[G(x)]^{r-1} g(x) d x
\end{aligned}
$$

Taking the derivative, we get (2.26) and we obtain, after integration, (2.2). Thus (2.28) implies (2.2).

\section{Special case}

Return to (2.28), then put $l=-1, v=1$, we get

$$
\mu_{r \mid r+1}=\varphi(y)+\frac{1}{b c r}\left\{a E_{r \mid r+1}\left[V\left(X_{r: n}\right) \mid X_{r+1}=y\right]-b E_{r \mid r+1}\left[\varphi\left(X_{r n n}\right) V\left(X_{r n n}\right) \mid X_{r+1}=y\right],\right.
$$$$
\alpha<x<y<\beta,
$$

it is before doubly truncated case.

\section{Theorem 7}

Referring to (1.5), (1.7) and (2.2), then

$$
\begin{aligned}
& \mu_{s \mid r}=\mu_{s-|| r}-\frac{l}{b c(n-s+1)} \\
& \left\{b E_{s \mid r}\left[\varphi\left(X_{s, n} x\right) N\left(X_{s: n}\right) \mid X_{r: n}=x\right]-a E_{s \mid r}\left[N\left(X_{s: n}\right) \mid X_{r \cdot n}=x\right]\right\}
\end{aligned},
$$

where $N(y)$ is defined in (2.17).

\section{Proof} have

As before in Theorem (3), compensation for (2.26) in (2.18), we

$$
\begin{aligned}
& \mu_{s \mid r}=\mu_{s-1 \mid}-\frac{(n-r) !}{b c(s-r-1) !(n-s+1) ![1-G(x)]^{n-r}} \int_{x}^{y}(b \varphi(y)-a) N(y)[G(y)-G(x)]^{s-r-1} \\
& \times[1-G(y)]^{n-s} g(y) d y=\mu_{s-1 \mid}-\frac{l}{b c(n-s+1)}\left\{\frac{1}{c} \int_{x}^{1}(b \varphi(y)-a) N(y) g_{s \mid r}(y \mid x) d y\right.
\end{aligned}
$$

After simplification, we get (2.31). Then (2.2) implies (2.31).

\section{Theorem 8}

Referring to (1.5), (1.7), then (2.2) if and only if

$$
\begin{aligned}
& \mu_{r+1 \mid r}=\varphi(x)+\frac{l}{b c(n-r)} \\
& \left\{b E_{r+1 \mid r}\left[\varphi\left(X_{r+1: n}\right) N\left(X_{r+1: n}\right) \mid X_{r: n}=x\right]-a E_{r+1 \mid r}\left[N\left(X_{r+1: n}\right) \mid X_{r: n}=x\right]\right\}
\end{aligned}
$$

where $N(y)$ is defined in (2.17).

\section{Proof}

As before in Theorem (4), compensation for (2.26) in (2.23), we have

$$
\begin{aligned}
& \mu_{r+1 \mid r}=\varphi(x)+\frac{l}{b c[1-G(x)]^{n-r}} \\
& \int_{x}^{\gamma}[b \varphi(y)-a] N(y)[1-G(y)]^{n-r-1} g(y) d y
\end{aligned}
$$

Then, we obtain (2.35). Thus (2.2) implies (2.33). Now from (1.7) and (2.33) we get

\begin{tabular}{|c|c|c|c|c|}
\hline Name & {$[I G(x)+v]$} & $\phi(x)$ & $(I, v)$ & $(a, b, c)$ \\
\hline Weibull & $\left(e^{-\theta \varepsilon^{p}}-e^{-\theta \gamma^{p}}, e^{-\theta \varepsilon^{p}}\right)$ & $\begin{array}{l}e^{-x^{p}} \\
e^{-\theta x^{p}}\end{array}$ & $e^{-\theta x^{p}}$ & $(-1,0, \theta)$ \\
\hline Power function & $\begin{array}{l}1-\theta^{-p} x^{p} \\
\alpha \leq \varepsilon<x<\gamma \leq \beta, \varepsilon=0, y \rightarrow \infty\end{array}$ & $\underbrace{\left(\frac{x}{\theta}\right)^{p}}_{X^{p}}$ & $\left(\theta^{-p}\left(\mathcal{\varepsilon}^{p}+\gamma^{p}\right), 1-\theta^{-p} \mathcal{E}^{p}\right)$ & $\begin{array}{l}(1,1,1) \\
\left(\theta^{-p}, 1,1\right)\end{array}$ \\
\hline Rayleigh & $\begin{array}{l}e^{-\theta x^{2}} ; \alpha \leq \varepsilon<x<\gamma \leq \beta \\
\varepsilon=0, \gamma \rightarrow \infty\end{array}$ & $e^{-x^{2}}$ & $\left(e^{-\theta \gamma^{2}}+e^{-\theta \varepsilon^{2}}, e^{-\theta \gamma^{2}}\right)$ & $(-1,0, \theta)$ \\
\hline Inverse Weibull & $\begin{array}{l}e^{-\theta x^{-p}} ; \alpha \leq \varepsilon<x<\gamma \leq \beta, \\
\varepsilon=0, \gamma \rightarrow \infty\end{array}$ & $e^{-\theta x^{-p}}$ & $\left(e^{-\theta \gamma^{-p}}+e^{-\theta \varepsilon^{-p}}, e^{-\theta \varepsilon^{-p}}\right)$ & $(0,-1,1)$ \\
\hline
\end{tabular}

$$
\begin{aligned}
& \frac{(n-r)}{[1-G(x)]^{n-r}} \int_{x}^{\gamma} \varphi(y)[1-G(y)]^{n-r-1} g(y) d y=\varphi(x)+\frac{l}{c[1-G(x)]^{n-r}} \int_{x}^{y} \varphi(y) N(y) \\
& \times[1-G(y)]^{n-r-1} g(y) d y-\frac{a l}{b c[1-G(x)]^{n-r}} \int_{x}^{\gamma} N(y)[1-G(y)]^{n-r-1} g(y) d y
\end{aligned}
$$

Taking the derivative with respect to $\mathrm{x}$, we obtain (2.26), and integrate it we have (2.2), thus (2.33) implies (2.2).

Hence, the Theorem is proved. Special caseReturn to (2.33), then put $l=-1, v=1$, we get

$$
\begin{aligned}
& \mu_{r+1 \mid r}=\varphi(x)-\frac{1}{b c(n-r)} \\
& \left\{b E_{r+1 \mid r}[\varphi(Y) \mid x]-a\right\}, \alpha<x<y<\beta
\end{aligned}
$$

it is before doubly truncated case (Table 2).

\section{Theorem 9}

Referring to (1.6), (1.7) and (2.3), then

$$
\mu_{r \mid s}=\mu_{r+1 \mid s}-\frac{l}{a c r}\left\{(b-v) E_{r \mid s}\left[e^{c \varphi\left(X_{r: n}\right)} \mid X_{r: s}=y\right]-a\right\}
$$

Table 2: Example of $G(x)=\frac{1}{l}\left\{[b-a \varphi(x)]^{c}-v\right\}$ distributions. 
Citation: Shawky Al, Badr MM (2016) Characterizations of Certain Doubly Truncated Distribution Based on Order Statistics. J Appl Computat Math 5: 329. doi: 10.4172/2168-9679.1000329

Page 5 of 6

\begin{tabular}{|l|l|l|l|}
\hline Name & {$[\mathbf{I G}(\boldsymbol{x})+\boldsymbol{v}]$} & $\boldsymbol{\phi}(\boldsymbol{x})$ & $(\boldsymbol{I}, \boldsymbol{v})$ \\
\hline Power distribution & $1-\theta^{-p} X^{p}, \alpha \leq \varepsilon<x<\gamma \leq \beta, \varepsilon=0, \gamma=\theta$ & $\operatorname{Ln}\left[1-\theta^{-p} X^{p}\right]$ & $\left(\theta^{-p}\left(\varepsilon^{p}+\gamma^{p}\right), 1-\theta^{-p} \varepsilon^{p}\right)$ \\
\hline Weibull & $e^{-\theta x p}, \alpha \leq \varepsilon<x<\gamma \leq \beta, \varepsilon=0, \gamma \rightarrow \infty$ & $\theta x^{p}$ & $\left(e^{-\theta \gamma^{p}}+e^{-\theta \varepsilon^{p}}, e^{-\theta \varepsilon^{p}}\right)$ \\
\hline Burr & $\left(1+\theta x^{p}\right)^{-\gamma}, \alpha \leq \varepsilon<x<\gamma \leq \beta, \varepsilon=0, \gamma \rightarrow \infty$ & $(1,0,-1)$ & $(-1,0,1)$ \\
\hline Inverse Weibull & $e^{-\theta x^{-p}}, \alpha \leq \varepsilon<x<\gamma \leq \beta, \varepsilon=0, \gamma \rightarrow \infty$ & $\ln \left[1+\theta x^{p}\right]$ & $\left.\left.\left(\theta \gamma^{-p}\right)+\theta^{\varepsilon p}+1\right), 1+\theta^{\varepsilon p}\right)$ \\
& & $(-1,0, \gamma)$ & $\left(e^{-\theta \gamma^{p}}+e^{-\theta \varepsilon^{p}}, e^{-\theta \varepsilon^{-p}}\right)$ \\
\hline
\end{tabular}

Table 3: Example of $G(x)=\frac{1}{l}\left\{b-a e^{-c \varphi(x)}-v\right\}$ distributions.

\section{Proof}

As given in Theorems (1) and (5), , differentiate (2.3) with respect to $\mathrm{x}$, we have

$$
\varphi(x)=\frac{l}{a c} e^{c \varphi(x)} g(x) .
$$

Compensation for (2.37) in (2.7), we get

$$
\mu_{r \mid s}=\mu_{r+1 \mid s}-\frac{1}{a c r} \int_{\varepsilon}^{y}\left[(b-v) e^{c \varphi(x)}-a\right] g_{r \mid s}(x \mid y) d x,
$$

which gives (2.36). Thus, (2.3) implies (2.36).

\section{Theorem 10}

Referring to (1.6) and (1.7), then (2.3) if and only if

$$
\begin{aligned}
& \mu_{r \mid r+1}=\varphi(y)-\frac{1}{a c r}\left\{(b-v) E_{r \mid r+1}\left[e^{c \varphi\left(X_{r n n}\right)} \mid X_{r+1: n}=y\right]-a\right\}, \\
& \text { Proof }
\end{aligned}
$$

As given previously of Theorems (2) and (6), substituting from (2.26) in (2.11), we have

$$
\mu_{r \mid r+1}=\varphi(y)-\frac{1}{a c r} \int_{\varepsilon}^{y} e^{c \varphi(x)}\left[b-v-a e^{-c \varphi(x)}\right] g_{r \mid r+1}(x \mid y) d x
$$

After some simplification, we get (2.36). Then (2.3) implies (2.36). Now from (1.7) and (2.36), we obtain

$$
\begin{aligned}
& r \int_{\varepsilon}^{y} \varphi(x)[G(x)]^{r-1} g(x) d x=[G(y)]^{r} \\
& {\left[\varphi(y)-\frac{(b-v)}{a c r} \int_{\varepsilon}^{y} \frac{r[G(x)]^{r-1} e^{c \varphi(x)} g(x)}{[G(y)]^{r}} d x+\frac{1}{c r}\right]}
\end{aligned}
$$

Taking the derivative with respect to $\mathrm{y}$, we get

$$
\frac{\lg (y)}{l \mathrm{G}(y)}=\frac{a c e^{-c \varphi(y)} \varphi(y)}{b-v-a e^{-c \varphi(y)}} \text {. }
$$

Integrate (2.41), we obtain (2.3).

\section{Special case}

Return to (2.36), then put $l=-1, v=1$, we get

$$
\mu_{r \mid r+1}=\varphi(y)-\frac{1}{a c r}\left\{(b-1) E_{r \mid r+1}\left[e^{c \varphi\left(X_{r n n}\right)} \mid X_{r+1: n}=y\right]-a\right\}, \alpha<x<y<\beta
$$

it is before doubly truncated case.

\section{Theorem 11}

Referring to (1.5), (1.7) and (2.3), then

$$
\mu_{s \mid r}=\mu_{s-1 \mid r}-\frac{l}{a c(n-s+1)}\left\{(l-b+v) E_{s \mid r}\left[e^{c \varphi\left(x_{s n n}\right)} \mid X_{r: n}=x\right]+a .\right.
$$

\section{Proof}

As previously in Theorems (3) and (7), from (2.37) in (2.18), we have

$$
\mu_{s \mid r}=\mu_{s-1 \mid r}-\frac{1}{a c(n-s+1)} \int_{x}^{\gamma}\left[(l-b+v) e^{c \varphi(y)}+a\right] g_{s \mid r}(y \mid x) d y,
$$
which gives (2.42).

\section{Theorem 12}

Referring to (1.5) and (1.7), then (2.3) if and only if

$$
\mu_{r+1 \mid r}=\varphi(x)+\frac{(l-b+v)}{a c(n-r)} E_{r+1 \mid r}\left[e^{c \varphi\left(X_{r+1 \mid n}\right)} \mid X_{r: n}=x\right]+\frac{1}{c(n-r)} . \text { (2. }
$$

Proof

Similarly as given in Theorems (4) and (8), we easily prove it.

\section{Special case}

Return to (2.43), then put $l=-1, v=1$, we get

$$
\mu_{r+1 \mid r}=\varphi(x)-\frac{b}{a c(n-r)} E_{r+1 \mid r}\left[e^{c \varphi\left(X_{r+t+n}\right)} \mid X_{r: n}=x\right]+\frac{1}{c(n-r)}, \alpha<x<y<\beta .
$$

The relation before doubly truncated case (Table 3 ).

\section{Conclusion}

It was obtained recurrence relations based on order statistics without truncated and doubly truncated, and have been getting function of various distributions new by using certain parameters.

\section{Acknowledgement}

This paper was founded by the Deanship of Scientific Research (DSR), King Abdulaziz University,Jeddah, under grant No. (65-363-D1431).The authors therefore, acknowledge with thanks DSR technical and financial support.

\section{References}

1. David HA (1981) Order Statistics. (2ndedn) John Wiley, New York.

2. Balakrishnan N, Cohen AC (1991) Order Statistics and Inference: Estimation Methods. Academic Press, San Diego.

3. Arnold BC, Balakrishnan N, Nagaraja HN (1992) A first Course in order Statistics. John Wiley, New York.

4. David HA (1995) On recurrence relations for order statistics. Statistics and Probability Letters 24: 133-138.

5. David HA, Nagaraja HN (2003) Order Statistics. (3rdedn) John Wiley, New York.

6. Mahmoud MAW, Sultan KS, Amer SM (2003) Order statistics from inverse Weibull distribution and associated inference. Comput Statist Data Analysis 42: 49-163.

7. Mahmoud MAW, Sultan KS, Amer SM (2003) Order statistics from inverse Weibull distribution and characterizations. Metron LXI 3: 389-401.

8. Balakrishnan N, Sultan KS (1998) Recurrence relations and identities for moments of order statistics. In: Balakrishnan N and Rao CR (eds.) Handbook of statistics16: 149-228.

9. El-Din M, Mahmoud MM, Abu-Youssef MAW, Sultan SE (1997) Order statistics from the doubly truncated linear-exponential distribution and its characterizations. Commun Statist- Simul Comput 26: 281-290.

10. Abu-Youssef SE (2003) On characterization of certain distribution of record values. Applied Mathematics and Computation 145: 445-450. 
Citation: Shawky AI, Badr MM (2016) Characterizations of Certain Doubly Truncated Distribution Based on Order Statistics. J Appl Computat Math 5: 329. doi: 10.4172/2168-9679.1000329

Page 6 of 6

11. Abd-El-Mougod GA (2005) On Record Values and Its Applications. Sohag University, Egypt.

12. Shawky Al, Abu-Zinadah $\mathrm{HH}$ (2008) General recurrence relations and characterizations of certain distributions based on order statistics. Journal of Statistical Theory and Applications 7: 93-117.

13. Shawky Al, Bakoban RA (2009) Conditional expectation of certain distributions of record values. Int Journal of Math Analysis 3: 829-838.

14. Pushkarna N, Saran J, Tiwari R (2012) Recurrence relations for higher moments of order statistics from doubly truncated exponential distribution. International Mathematical Forum, 7(4), 193-201.
15. Joshi PC (1979) A note on the moments of order statistics from doubly truncated exponential distribution. Annals of the Institute of Statistical Mathematics 31 : 321-324.

16. Balakrishnan N, Joshi PC (1984) Product moments of order statistics from the doubly truncated exponential distribution. Naval Research Logistics Quarterly: 31: $27-31$

17. Khan AH, Ali MM (1987) Characterization of probability distributions through higher order gap. Communications in Statistics-Theory and Methods 16 1281-1287.

18. Ahmad AA (2001) Moments of order statistics from doubly truncated continuous distribution. Statistics 35: 479-494. 